\title{
Human gut microbiota evaluation and comparation after the separate or combined antibiotics exposure using the simulator of human intestinal microbial ecosystem (SHIME)
}

Lei Liu

Nankai University

Ranjit Das

Nankai University

Pengcheng Suo

Nanjing University of Information Science and Technology

Huai Lin

Nankai University

Hongmei Qi

Nankai University

Siyi Wang

Nankai University

Xiaojun Zuo ( $\nabla$ z850108xj@163.com )

Nanjing University of Information Science and Technology https://orcid.org/0000-0001-6823-1962

\section{Research}

Keywords: Amoxicillin (AMX), Combination effects, Human disease-related pathways, Polymyxin E (POL), Simulator of the human intestinal microbial ecosystem (SHIME)

Posted Date: June 10th, 2020

DOI: https://doi.org/10.21203/rs.3.rs-34434/v1

License: (c) (1) This work is licensed under a Creative Commons Attribution 4.0 International License. Read Full License 


\section{Abstract}

Background A cocktail of drugs is an emerging toxic contaminant that has potential public health risk worldwide, which also would cause human intestinal microbial disorder and develop multiple human diseases. However, to date, the combination effects of antibiotics cocktail on human intestinal microbiota dysbiosis and related health risk are not fully understood. Therefore, for the first time, this study evaluated and compared the in vitro ability of amoxicillin (AMX) and polymyxin $E(P O L)$ used separately or combined on antibiotic resistance genes (ARGs) as well as human disease-related pathways in the simulated human gut.

Results This study indicated that the combination exposure of POL with AMX reduced the occurrence of drug resistance in the gut microbiota caused by single antibiotic treatment. However, in comparison with the separate use of AMX and POL, the combined treatment exhibited a significantly higher ability to increase the human disease-related pathways. The combination effects on genetic level might attribute to microbiota shift, as co-occurrence patterns suggested that Bifidobacterium attributed to ARGs increasing in the POL treatment group and Enterobacter played a crucial role in human disease-related pathways enrichment after combination treatment.

Conclusion These results may open up new perspectives for assessing the direct effects of combination antibiotics on the intestinal microbiota. These suggested side-effects should be considered for a combination of antibiotics prescription.

\section{Background}

The antibiotic therapies have been demonstrated paramount importance in the treatment of bacterial infections since its discovery in the 1940s. Nowadays the extensively used antibiotics are considered as toxic emerging contaminants, which pose severe threats to environmental ecosystems [1]. Humans are directly and indirectly exposed to different antibiotics cocktail through inhalation, ingestion of drugs, drinking water, and foods, and their risk assessments have attracted more attention, recently [2-4]. As a result, antibiotics may progressively enter into the human gastrointestinal tract and produce a large variety of antibiotic-resistant gut-micro flora [5]. In our previous research, it was found that both human and veterinary antibiotics were mostly detected in the gut of the Chinese population [6]. The stable intestinal microbial ecosystem has been demonstrated not only to provide essential nutrients for human health but also to modulate the immune function by protecting infectious pathogens $[7,8]$. However, exposure to antibiotics cocktail may lead to the disrupt of the stable ecosystem and promote the spread of antibiotic-resistant bacteria (ARB) and antibiotic resistance genes (ARGs) in the human gut, which may limit the treatment efficiency and resulting from the chronic and relapsing infectious diseases [9-11]. And loop forever, host-associated fecal with antibiotic residues as well as ARB and ARGs would contaminate the environment [12-14]. 
Amoxicillin (AMX) is a broad-spectrum oral penicillin-type beta-lactam antibiotic, which kills bacteria by interfering with the synthesis of bacterial cell wall peptidoglycan layers $[15,16]$. Besides, polymyxin $E$ (POL) is known as a colistin drug, a kind of cationic peptides that binds to anionic lipopolysaccharide of the gram-negative bacterial cell membrane and displaces calcium and magnesium ions from the outer cell, which further leads to change of cell membrane permeability and ultimately cell death [17]. Over the last several years, the effects of AMX or POL on the human intestinal microbiota have been extensively studied [18-24]. However, only a few research papers have reported their combination effects on intestinal microbial ecosystem by animal models, which would not reflect the actual influences of antibiotics on human gut microbiota [25-27]. Furthermore, these in vivo studies didn't reveal the direct effects of antibiotics on intestinal microbiota because of the disturbances of neurohumoral regulation, the individual differences, dietary habits, and the physiological status. The simulator of the human intestinal microbial ecosystem (SHIME) model is known to be a useful tool for in vitro studies as (i) interactions between the microbiota; and (ii) the effects of prebiotics and other compounds on the microbial communities and metabolic activities [28]. To the best of our knowledge, there are only a few researches that added the antibiotics into this in vitro model, which mainly focused on the benefit of the mucosal environment, high-fiber diets, probiotic, and propionate-producing consortium in human intestinal microbiota [29-32]. Moreover, the combination effect of antibiotics cocktail on human intestinal microbiota dysbiosis and related health risk is not fully understood.

Therefore, this study, for the first time, evaluated and compared the in vitro ability of AMX and POL (used separately or combined) on ARGs as well as human disease-related pathways in the simulated human gut. Considering the reasonable dosage of AMX and POL for adult human study are about 750 to 1500 $\mathrm{mg} /$ day and $1.5-4 \times 10^{6} \mathrm{IU} /$ day respectively, and only half volume of the adult gut can be simulated in used SHIME model, $600 \mathrm{mg} /$ day of AMX and $1.5 \times 10^{6} \mathrm{IU} /$ day of POL were used to examine its impacts on human gut microbiota and associated functional pathways [18, 20, 23, 24]. In this study, the composition of human intestinal microbiota was analyzed by $16 \mathrm{~S}$ rRNA gene high-throughput sequencing, the human disease-related pathways were predicted by functional predictions and the ARGs were quantified by high-throughput quantitative PCR (HT-qPCR). This study achieved a systematic investigation and precise understanding of the direct effects of AMX and POL on the intestinal microbiota, which may be valuable for directing future work.

\section{Results}

\section{ARGs}

There was a total of three batch experiments conducted, and we collected six different group samples (groups A_Con, A_AMX, P_Con, P_POL, Comb_Con, and Comb_AP, each group contain six samples from two-time points and three colon reactors, shown in Fig. 1). In this study, a total of 37 targets ARGs were detected from six different group samples using a high-throughput-qPCR (HT-qPCR) technique. The heatmap showed that the relative abundances of ARGs such as aminoglycoside, beta-lactam, multidrug, and tetracycline resistance genes were noticeably higher in the group P_POL than the group P_Con, while 
the difference were not comparable between groups Comb_Con and Comb_AP (Fig. 2a). For instance, log relative abundance increment of aac6ib (aminoglycoside) was about 1.7 log units after POL treatment than the control (P_POLA1 vs. P_ConA2), while b/2b_ampc (beta_lactam), mexf (multidrug), and tetra (tetracycline) were about 2.0, 2.4, and 1.9 log units, respectively. However, the increments of log relative abundance of the above mentioned ARGs were just about 0-0.9 log units after AMX treatment than in control (A_AMXA1 vs. A_ConA2), and 0-0.5 log units for combination treatment (Comb_APA1 vs. Comb_ConA2). Additionally, during PCA analysis, the component one explained $29.3 \%$ of the variance, and the component two exhibited $15.2 \%$ of the variance (Fig. $2 \mathrm{~b}$ ). These data clearly displayed the differences in ARGs abundances of bacteria community between the POL treatment and the control group (P_POL vs. P_Con), while a combination of antibiotics treatment attenuated the discrepancy (Comb_AP vs. Comb_Con).

\section{Human disease-related pathways}

The metagenomics study of the 16S rRNA gene sequence by PICRUSt revealed the gene numbers of human disease-related functional pathways in the bacterial communities of the six groups, and the genes were presented in the heatmap (Fig. 3a). More importantly, the heatmap showed the gene numbers of human disease-related pathways, including cancer, drug resistance, endocrine and metabolic diseases, infectious diseases, and neurosurgery diseases, which were more abundant in AMX treatment group than that in control group, and the distinction was especially more apparent between groups Comb_Con and Comb_AP. For instance, the gene numbers of colorectal cancer, viral myocarditis, hepatitis B, and toxoplasmosis were about 5 times enriched after AMX treatment than in the control (A_AMXA2 vs. A_ConA2). Moreover, the gene numbers of these human disease-related pathways were about 8 times enriched after POL treatment than in control (P_POLA2 vs. P_ConA2), while gene numbers were about 51 times enriched for combination treatment (Comb_APA2 vs. Comb_ConA2). The first (PC1) and second principal component (PC2) explained $64.8 \%$ and $10.7 \%$ of the total variance, respectively (Fig. $3 \mathrm{~b}$ ), and the distinguished feature indicated that the combination of antibiotics treatment had the greatest impact (Comb_AP vs Comb_Con) on the human disease-related pathways of the bacterial community.

\section{Microbiota community composition}

In this study, the effects of antibiotics on gut microbial communities' composition were also investigated. Based on the 16S rRNA gene sequence analysis, the most abundant taxonomic groups assigned at the phylum level were Proteobacteria (14.6-97.0\%), Firmicutes (2.4-47.5\%) and Bacteroidetes (0.1-47.2\%) (a total of 62.1-100.0\%), followed by Synergistetes, Actinobacteria, and Verrucornicrobia (Fig. S1). Moreover, an obvious decrease of Bacteroidetes (from 7.5-29.8\% to 0.0-3.2\% except for P_POLD1), and increase of Actinobacteria (from $0.0-0.4 \%$ to $0.2-37.3 \%$ ) were also seen after POL treatment (Fig. S1b).

At the genus level, the AMX-treated subjects were shown to be substantially overgrown by Enterobacter (from 1.3-2.1\% to 5.6-38.5\%) and Bacteroides (from 7.8-11.6\% to 7.8-23.0\%), while the percentage of Escherichia-Shigella (from 20.7-70.0\% to 4.0-14.6\%) was decreased (Fig. 3). However, Pseudomonas (from 1.1-9.0\% to $0.3-57.3 \%$ ) and Bifidobacterium (from $0.0-0.3 \%$ to $0.1-35.1 \%$ ) were bloomed in the POL 
treatment group, while the percentage of Escherichia-Shigella (from 33.5-39.6\% to 2.6-24.2\%) was decreased. Moreover, the combination treatment (Comb_AP vs Comb_Con) caused enrichment of Enterobacter (from 1.1-6.2\% to 5.8-49.6\%) and Pseudomonas (from 0.0-4.7\% to $0.1-29.9 \%$ ), and reduction of Escherichia-Shigella (from 2.6-40.7\% to 0.1-5.0\%). The linear discriminant analysis effect size (LEfSe) comparison analysis between the six groups is shown in Fig. S2. LEfSe analysis indicated that AMX was significantly decreased in several genera, including Escherichia-Shigella (LSD = 5.32), Cloacibacillus (LSD $=4.58)$, and Megasphaera (LSD = 4.41). The changes were accompanied by the increase in the relative abundances of Bacteroides (LSD $=4.87)$, Klebsiella (LSD $=4.57)$, Lachnoclostridium (LSD $=4.43)$, and Acinetobacter (LSD = 4.12), which were also consistent with the above gut microbial communities' composition results.

\section{Microbiota diversity}

Meanwhile, the fecal microbiota of alpha diversity was assessed. The taxon richness (Chao1 index), evenness (Simpson index), and diversity (Shannon index) are shown in Fig. S3. Compared with the control group (A_Con) the decline of microbial richness (Chao1, $P=0.777$ ), evenness (Simpson, $P=$ 0.046), and increase of diversity (Shannon, $P=0.030$ ) were observed in the samples after AMX treatment; however opposite changes were occurred in the groups Comb_Con and Comb_AP (Chao1, P $=0.495$; Simpson, $P=0.010$; Shannon, $P=0.022$ ). As for the $P O L$ treatment group, no obvious change was found in alpha diversity (Chao1, $P=0.496$; Simpson, $P=0.727$; Shannon, $P=0.291$ ).

Besides, the beta diversity of the microbiota communities and weighted UniFrac distance was affected by antibiotics treatment. As shown in Fig. 3d, three control groups were clustered together in PCA analyses. The beta diversity results showed that all the samples collected after a single antibiotic or combination treatment differed from the control group. Meanwhile, weighted UniFrac distances between a single antibiotic treatment group and the control group (A_AMX vs. A_Con, P_POL vs. P_Con) were all significantly higher $(P<0.001$, T-test) than that within the control group (A_Con vs. A_Con, P_Con vs. P_Con), and that between the combination group Comb_AP and the control group Comb_Con were slightly higher ( $P<0.01$, T-test) than that within the control (Fig. S4).

\section{Correlations between microbial taxa and human disease-related pathways or ARGs}

The network analysis of co-occurrence patterns between the microbial taxa and the ARG subtypes is shown in Fig. 5a. It was seen that Bifidobacterium (significantly enriched bacteria after POL treatment) was positively associated with aminoglycoside, macrolide-lincosamide-streptogramin B (MLSB), multidrug, and tetracycline resistance genes. For example, the correlation coefficient of Bifidobacterium with qacedelta1 and tetw was about $0.8(P<0.001)$, and the correlation coefficient with aac3iia and mpha was about $0.7(P<0.001)$. Pseudomonas, another significantly increased bacterial genus in either POL treatment or combination treatment group, was also positively associated with several ARGs. For example, the strong correlations were found in Pseudomonas with mexf and b/1_ampc (about 0.8, $P<$ 0.001), and with tetpa and aac6ib (about 0.7, $P<0.001$ ). 
Fig. $5 \mathrm{~b}$ shows the results of co-occurrence patterns between the microbial taxa and human diseaserelated pathways. A very similar pattern of results was observed that significantly increased bacteria Enterobacter (after in either POL treatment or combination treatment group) was positively associated with almost all of those human disease-related pathways, such as cancer, drug resistance, endocrine, and metabolic diseases, infectious diseases, and neurosurgery diseases. Specifically, the correlation coefficient of Enterobacter with bladder cancer, pertussis, and Salmonella infection was about $0.95(P<$ $0.001)$ and the correlation coefficient with Prion diseases and cationic antimicrobial peptide (CAMP) resistance was $0.94(P<0.001)$.

\section{Discussion}

After two weeks of SHIME operation, the microbiota's composition and diversity were stabilized, and most predominant phyla in the gut microbiome were Bacteriodetes, Proteobacteria, Synergistetes, and Firmicutes, which were also previously demonstrated by Yu's group [33]. \{Yu, 2016 \#1;Li, 2015 \#3\} These predominant phyla had also been observed in the fecal samples of humans and animals [34, 35]. As mentioned above, a large body of data showed that stabilized microbiota might be disturbed by antibiotics exposure, resulting in chronic and relapsing infections [9-11]. The effects of AMX or POL on the human intestinal microbiota have been extensively studied [18-24]. However, the combination effect of antibiotics cocktail on human intestinal microbiota dysbiosis and related health risk is not fully understood. Hence, this study for the first time evaluated and compared the in vitro ability of AMX and POL (used separately or combined) on ARGs as well as human disease-related pathways in the simulated human gut. As the bacteria-killing mechanism for AMX is interfering synthesis of the bacterial cell wall peptidoglycan $[15,16]$, whereas $\mathrm{POL}$ is binding to the anionic lipopolysaccharide of the gram-negative bacterial cell membrane [17]. Therefore, it is supposed that the combination of AMX and POL can cause a synergistic effect on human intestinal microbiota.

\section{Combination treatment reduced occurrence of drug resistance development}

As mentioned earlier, exposure to antibiotics may promote the spread of ARB and ARGs in the human gut, which may limit the treatment efficiency and resulting in chronic and relapsing infections [9-11]. For instance, ARGs from such classes, including aminoglycosides, beta-lactams, and tetracycline have been confirmed to enrich in human gut microbiota following administration of AMX-clavulanic acid for one week [36], while patients who have taken AMX lower the relative abundance of ARGs from $0.81 \%$ to $0.14 \%$ [37]. The controversial conclusions in their results may attribute to the subjects, antibiotics administration and, the statistical approach of ARGs varied widely between studies. The minor effect of AMX on ARGs found in our study was not totally in agreement with the results. Barraud and associates discovered that AMX exposure to women during labor significantly selected bla (TEM)-positive Enterobacteria in their gut microbiota as well as their children's, indicating ARB would cumulate across generation [38]. Similarly, $\mathrm{POL}$ was suggested to cause the selection of antimicrobial-resistant bacteria and the exchange of ARGs through horizontal gene transfer (HGT) between bacterial species [23]. Buelow and associates also confirmed that cefotaxime, POL, tobramycin, and amphotericin B combination treatment would select the 
antibiotic-resistant bacteria and aminoglycoside resistance genes such as $a p h(2 ")-\mathrm{lb}$ and the aadE-like gene [39]. Furthermore, the increment of relative log abundance of ARGs such as aac6ib (aminoglycoside) caused by POL treatment that showed in our research was also consistent with the previous results.

The extensive use and misuse of antibiotics are known as contributors to the development of antibiotic resistance, which is a recent threat to global health. Antibiotics combination therapy is one of the essential ways that can prevent the development of antimicrobial resistance [40-42]. For instance, the addition of an aminoglycoside to a beta-lactam therapy regimen has been suggested to have a beneficial effect in delaying or preventing the development of antimicrobial resistance [43]. Combination therapy also suppresses or minimizes the degree of resistance of daptomycin-resistant viridans group streptococci compared with daptomycin monotherapy [44]. This study, for the first time, revealed that the combination of $\mathrm{AMX}$ and $\mathrm{POL}$ treatment reduced the occurrence of drug resistance development, which was consistent with the above findings. However, the combination therapy with beta-lactam ceftazidime and the fluoroquinolone ciprofloxacin selected for mutants that displayed broad-spectrum resistance, leading to decreased susceptibility to the combination of drugs applied as well as to unrelated antibiotic classes [45]. Therefore, the increased risk of selecting for broad-spectrum resistance antibiotic combinations should also be considered before being implemented in the clinical settings.

\section{Combination treatment promote the increase of the human disease related genes}

Antibiotics have been widely used for the prevention and treatment of diseases to humans. For instance, AMX is effective in resistant pneumococcal and Helicobacter pylori eradication therapy $[19,46]$. Oral POL contributes to intestinal eradication of multidrug-resistant Enterobacteriaceae, ESBL-producing Escherichia coli, and Klebsiella pneumoniae in immunocompromised patients [23, 24]. However, the facilitating clearance of targeted infections, antibiotic-induced disruption of the microbiota and immune homeostasis can lead to disease [47]. AMX had been found to promote the selection of human disease category genes, including drug resistance categories in intestinal microbiota, and the increased gene numbers of human disease-related functional pathways shown in our results were in agreement with this [48]. Some studies demonstrated that AMX increased the beta-lactam resistance and antibiotic-resistant bacteria in the gut $[19,49]$. Other papers reported $A M X$ would activate innate immunity and cause infectious diseases such as urinary tract infections, diarrhea, and liver abscess [50-53]. AMX was also suggested to affect insulin sensitivity and increase succinate, monosaccharide, and oligosaccharide levels in the fecal samples, and prenatal exposure was also associated with the increase of childhood overweight risk [54-56]. AMX was even confirmed to increase the risk of colon cancer [57]. All the above might be explained by our findings that the human disease-related pathways, including cancer, drug resistance, endocrine, and metabolic diseases, infectious diseases were more abundant in the AMX treatment group. Similarly, POL suggested the effect of immune responses and induce disease incidence, including insulin-dependent diabetes, diarrhea, bacterial infections, and translocation using animal models [58-62]. 
A combination of antibiotic therapy is frequently used to treat severe infections, which would not only prevent the development of antimicrobial resistance but also improve the treatment efficacy [40-42]. For instance, a study confirmed that combination of colistin-polymyxin B or tigecycline with a carbapenem therapy is superior to monotherapy for carbapenemase-producing Klebsiella pneumoniae [63]. Besides, the combination of AMX and POL therapy would effectively attenuate bacterial endotoxin- and Shiga exotoxin-mediated cytotoxicity and reduce mortality from peritonitis [25,64]. However, excessive use of combinations should be avoided because it might be associated with increased risk for the toxicity and super-infections [41]. Combination therapy was also reported to cause a rise in infections triggered by multidrug-resistant gram-negative organisms [65]. Another paper revealed that a combination of an aminoglycoside with beta-lactam is associated with an increased risk for nephrotoxicity [66]. This study, for the first time, revealed that the combination of AMX and POL treatment promote the increase of the human disease-related genes such as colorectal cancer, viral myocarditis, hepatitis B, and toxoplasmosis. Therefore, these results might indicate that the side effects of combination therapy should be carefully considered.

\section{Combination effects on the genetic level attribute to microbiota shift}

The phenomena of microbiota shift shown in our research are identical to several in vivo studies on intestinal microbiota; however, Proteobacteria phylum such as Enterobacter and Bacteroides were most predominantly detected following $A M X$ administration $[52,67,68]$. Furthermore, the percentage of Escherichia-Shigella was decreased after POL treatment found in this study was further supported by earlier reports that confirmed the eradication ability of POL on Escherichia and its cytotoxicity [23,64]. Some studies have also discovered a trend toward increasing taxonomic diversity after AMX treatment and a significant effect on the beta diversity pursued by POL exposure [23,69]. Additionally, this study, for the first time, revealed that POL treatment caused a bloom of Pseudomonas and Bifidobacterium, while combination treatment (AMX and POL) caused the enrichment of Enterobacter and Pseudomonas. Bifidobacterium intrinsically carries ARGs such as ermx and tetw, and Pseudomonas possesses mexf, aada6, and blap1b, which make them inherently resistant to MLSB, tetracycline, aminoglycosides, and beta-lactam antibiotics, and consequently pose a significant therapeutic challenge [70-73]. All of the above findings supported the positive correlations between the abundances of Bifidobacterium and Pseudomonas with ARGs in our study. Significant Bifidobacterium shift discovered in this study also explained POL exposure caused more severe drug resistance development than combination treatment. Although combination treatment reduced the occurrence of drug resistance development produced by single POL treatment. However, the bloomed multidrug-resistant Pseudomonas was still an immense threat to human health because of its high virulence factor reported in many previous research papers [74-76].

Besides, Enterobacter was confirmed to increase after both AMX administration and combine exposure of antibiotics, and the combination treatment caused its more substantial enrichment. Although these bacteria are considered commensal in the human gut, fecal carriage of this opportunistic pathogen confirmed to be a severe risk factor for the infection [77-79]. The significant increase in opportunistic 
pathogen associated with human disease-related pathways of human intestinal microbiota following combination of AMX and POL treatment exhibited in this study have not been reported in details yet. More importantly, this study displayed a positive correlation between the opportunistic pathogen Enterobacter and human disease-related pathways, which suggested the bloom of this pathogen caused by antibiotics treatment that might contribute to human diseases. For instance, Enterobacter was reported to be related to human infectious diseases such as colitis, neonatal sepsis, and bloodstream infections [80-82]. Furthermore, some studies have also indicated that Enterobacter was enriched in cancer patients and related to cardiovascular diseases, endocrine and metabolic diseases, and neurosurgery diseases [83-87]. All of the findings, as mentioned above, were linked with positive correlations between the abundance of Enterobacter and human disease-related pathways. And this advocated that the enhanced effect of combination treatment on the increase of the pathways might attribute to apparent Enterobacter enrichment in our study. Therefore, the results of our research demonstrated a severe impact and a negative side-effect of combination antibiotics treatment that enriched pathogens related to health problems, which should be considered as a fundamental aspect of the cost-benefit equation for the antibiotics combination prescription.

\section{Perspectives}

The findings in this study suggested several numbers of opportunities for additional study. First, expansion of the analysis to incorporate multiple-omics approaches of the metagenome, metatranscriptome, and metabolome were not taken in this study, which attributed to the costs associated with sequencing and analysis. With multiple-omics data, the confirmed genome composition and expression of the gut microbiota would help us understand how antibiotics induced human intestinal microbiota dysbiosis and related disease. It is also of interest to inoculate the microbiota mixture from several patients to discover the different effects of antibiotics between healthy and sick individuals. However, it is difficult to obtain such samples, and the SHIME operation cost is very high. Further in vivo studies aimed to verify whether the findings in this in vitro study reflect the reality, which will provide new insights to measure how antibiotic affects the gut microbiota and the associated disease. Moreover, the combination antibiotics' exposure was confirmed to cause the synergistic effect on increasing the human disease-related genes, and their prescription should be considered as an essential aspect of the risk assessment.

\section{Conclusions}

In this study, a combination of POL and AMX was confirmed to reduce the occurrence of drug resistance in the gut microbiota caused by single antibiotic treatment. However, the results also revealed that combination treatment would promote the increase of the disease-related pathways compared with the separate use of AMX or POL. Combination effects on the genetic level might attribute to microbiota shift, which were explained well by the phenomenon that Bifidobacterium was positively associated with the ARGs and Enterobacter was positively related to the human disease-related pathways. These results might be valuable to direct the future work and opened up new perspectives to address the direct effects 
of combine antibiotics on the intestinal microbiota. These suggested side-effects should be considered for a combination of antibiotics prescription.

\section{Methods}

\section{Antibiotics treatment and samples collection}

In this study, the SHIME was constructed using five double-jacketed reactors designated as the stomach, small intestine, ascending colon, transverse colon, and descending colon, respectively (Fig. 1). The last three reactors were inoculated with a mixture of fecal microbiota from one healthy adult volunteer, who did not suffer by any gastrointestinal disease or take antibiotics in the past six months, on account of the differences between individuals may be alleviated by same culture condition [88]. The details of the SHIME system and the startup process are summarized in the Supplementary material.

There was a total of three batch experiments conducted in which each one maintaining for two weeks of stabilization and afterward one week for the addition of nutritional medium (groups A_Con, P_Con, and Comb_Con, each group contain six samples from two-time points and three colon reactors). Then the SHIME was exposed individually by AMX (600 mg/day; group A_AMX), POL ( $1.5 \times 10^{6} \mathrm{IU} /$ day; group P_POL), as well as combination of them $\left(600 \mathrm{mg} /\right.$ day of AMX and $1.5 \times 10^{6} \mathrm{IU} /$ day of POL; group Comb_AP) for one week in each batch experiment (Fig. 1). Each sample is a mixture of three samples collected at specific time intervals in a day. The samples were stored at $-80^{\circ} \mathrm{C}$ for further analyses.

\section{S rRNA gene sequencing and analysis}

Total DNA was extracted from the samples using the E.Z.N.A. stool DNA Kit (Omega, USA) according to the manufacturer's protocols. The V3-V4 region of the bacterial 16S rRNA gene was amplified by polymerase chain reaction (PCR). The raw reads of the sequences were deposited into the NCBI Sequence Read Archive (SRA) database under the accession number SRR11249954-11249989. The raw Illumina fastq files were de-multiplexed, quality-filtered, and analyzed using Quantitative Insights Into Microbial Ecology (QIIME) [89]. The 16S rRNA gene sequences were further taxonomically classified using the Ribosomal Database Project (RDP) classifier 2.0.1 [90].

The effects of antibiotics on alpha diversity, the taxon richness (Chao1 index), evenness (Simpson index), and diversity (Shannon index) were calculated for all the samples as previous did [91, 92]. Linear discriminant analysis effect size (LEfSe) was performed to determine bacterial taxa that were significantly differed between the six groups using the Galaxy application tool [93]. Functional predictions of microbial community were performed to visualize the distribution of human disease-related pathways in the six groups using Phylogenetic Investigation of Communities by Reconstruction of Unobserved States (PICRUSt) [94]. The accuracy of PICRUSt for the detection of more challenging functional groups was good (min. accuracy $=0.82$ ), suggesting that their inference of gene abundance across various types of functions was reliable, and PICRUSt predictions had high agreement with metagenome sample abundances across all body sites (Spearman $r=0.82, P<0.001$ ). These analyses were conducted by 
BioMarker Technology Co., Ltd (Beijing, China). The details of taxonomical classification, LEfSe analysis, and functional predictions are described in the Supplementary material.

\section{High-throughput quantitative PCR (HT-qPCR) and analysis}

High-throughput-qPCR reactions were performed using the Wafergen SmartChip Real-time PCR system as previous did [91, 92]. The reactions were conducted by Anhui MicroAnaly Gene Technologies Co., Ltd (Anhui, China). A total of 108 primer sets were chosen (Excel S1), which included 102 primer sets to target the almost all major classes of antibiotic resistance genes (ARGs) found in the microbiota of Chinese human gut [95], along with five mobile genetic elements (MGEs) and one 16S rRNA gene. The results were analyzed using the SmartChip qPCR Software. Data with multiple melting peaks or amplification beyond the range (0) were excluded and then screened with conditions that a threshold cycle (CT) must be $<31$, and positive samples should have three replicates simultaneously. The details of HT-qPCR analysis are described in the Supplementary material.

\section{Data analysis}

All the results were expressed as mean values and standard deviations. The statistical analysis was performed with SPSS 17.0 software (SPSS Inc., Chicago, III., U.S.A.). The T-test was conducted to compare the differences between the groups, and all the statistical tests were two-tailed. The statistical

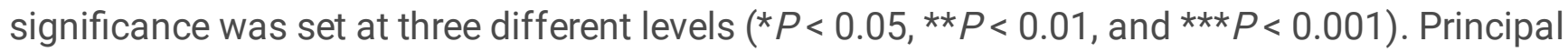
component analysis (PCA) of ARGs, human disease-related pathways, and OTUs were plotted by 'ggplot2', 'vegan', and 'corrplot' packages of R (version 3.5.1). Correlations between the microbiota and human disease-related pathways or ARGs were analyzed using the Spearman test in R with the 'vegan' package. The correlations between the pairs of variables were considered to be significant at $r>0.6$, and $P$ values were $<0.05$. The Gephi (V 0.9.1) software was used to visualize the bipartite network graphs using the Force Atlas algorithm.

\section{Additional Files}

Additional file 1: Supplementary information file. Supplementary Methods. Fig. S1. Composition of microbial community at phylum level in groups A_Con and A_AMX (a), P_Con and P_POL (b), Comb_Con and Comb_AP (c). Fig. S2. LDA score and cladogram of LEfSe comparison analysis between the six groups. The colored shading depicts bacterial taxa that were significantly higher in each group, as indicated. Selection of discriminative taxa between six groups were based on an LDA score cutoff of 4.0, and differences in the relative abundances of taxa were statistically determined based on a MannWhitney test at a significance level of 0.05. Fig. S3. Effect of antibiotic treatment on gut microbiota alpha diversity of the six groups ( ${ }^{\star \star *} p<0.001,{ }^{\star \star} p<0.01,{ }^{\star} p<0.05$ ). The Chao1 index (a) was used to calculate the community richness, Simpson index (b) was used to calculate the community evenness, and Shannon index (c) was used to calculate the community diversity. Fig. S4. Weighted UniFrac distance within control groups (A_Con vs A_Con, P_Con vs P_Con, Comb_Con vs Comb_Con) and between 


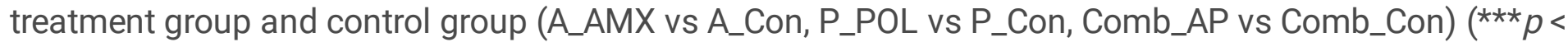
$0.001, * * p<0.01, * p<0.05)$.

Additional file 2: Excel S1 Primer sets of HT-qPCR reactions using Wafergen SmartChip Real-time PCR system.

\section{Declarations}

\section{Acknowledgments}

The authors express the sincerest thanks to Professor Bing Wu (School of the Environment in Nanjing University, Nanjing in China) for the guidance of SHIME, and BioMarker Technology Co., Ltd, and Anhui MicroAnaly Gene Technologies Co., Ltd for genome sequencing and analysis.

\section{Funding}

This work was supported by a grant from the project of 'Jiangsu Specially-Appointed Professor' (1421071901002).

\section{Availability of data and materials}

The datasets and scripts developed and generated in this manuscript are included within the manuscript and its supporting files.

\section{Consent for publication}

Not applicable.

\section{Authors' contributions}

Lei Liu: Methodology, Investigation, Formal analysis, Writing - original draft, Software. Ranjit Das: Writing - review \& editing. Pengcheng Suo: Investigation. Huai Lin: Investigation. Hongmei Qi: Investigation. Siyi Wang: Investigation. Xiaojun Zuo: Funding acquisition, Writing - review \& editing.

\section{Ethics approval and consent to participate}

The study was approved by the Biomedical Ethics Committees of Nankai University. The participant has given written, informed consent to understand the study's purpose, procedures, risks, benefits, and rights.

\section{Competing interest}

The authors declare that they have no competing interests. 


\section{References}

1. Ashbolt NJ, Amezquita A, Backhaus T, Borriello P, Brandt KK, Collignon P, et al. Human Health Risk Assessment (HHRA) for Environmental Development and Transfer of Antibiotic Resistance. Environ Health Perspect. 2013;121(9):993-1001.

2. Ben YJ, Fu CX, Hu M, Liu L, Wong MH, Zheng CM. Human health risk assessment of antibiotic resistance associated with antibiotic residues in the environment: A review. Environ Res. 2019;169:483-93.

3. Le Page G, Gunnarsson L, Snape J, Tyler CR. Integrating human and environmental health in antibiotic risk assessment: A critical analysis of protection goals, species sensitivity and antimicrobial resistance. Environ Int. 2017;109:155-69.

4. Nnadozie CE, Odume ON. Freshwater environments as reservoirs of antibiotic resistant bacteria and their role in the dissemination of antibiotic resistance genes. Environ Pollut. 2019;254.

5. Khan I, Yasir M, Azhar El, Kumosani T, Barbour EK, Bibi F, et al. Implication of gut microbiota in human health. CNS Neurol Disord Drug Targets. 2014;13(8):1325-33.

6. Wang Q, Duan YJ, Wang SP, Wang LT, Hou ZL, Cui YX, et al. Occurrence and distribution of clinical and veterinary antibiotics in the faeces of a Chinese population. J Hazard Mater. 2020;383:121129.

7. Cabreiro F, Au C, Leung KY, Vergara-Irigaray N, Cocheme HM, Noori T, et al. Metformin retards aging in C. elegans by altering microbial folate and methionine metabolism. Cell. 2013;153(1):228-39.

8. Qin JJ, Li RQ, Raes J, Arumugam M, Burgdorf KS, Manichanh C, et al. A human gut microbial gene catalogue established by metagenomic sequencing. Nature. 2010;464(7285):59-65.

9. Bengtsson-Palme J, Kristiansson E, Larsson DGJ. Environmental factors influencing the development and spread of antibiotic resistance. FEMS Microbiol Rev. 2018;42(1):68-80.

10. Stecher B, Maier L, Hardt WD. 'Blooming' in the gut: how dysbiosis might contribute to pathogen evolution. Nat Rev Microbiol. 2013;11(4):277-84.

11. Barraud O, Peyre M, Couve-Deacon E, Chainier D, Bahans C, Guigonis V, et al. Antibiotic resistance acquisition in the first week of life. Front Microbiol. 2018;9:1467.

12. Vadde KK, Feng Q, Wang J, McCarthy AJ, Sekar R. Next-generation sequencing reveals fecal contamination and potentially pathogenic bacteria in a major inflow river of Taihu Lake. Environ Pollut. 2019;254(Pt B):113108.

13. Somnark P, Chyerochana N, Mongkolsuk S, Sirikanchana K. Performance evaluation of Bacteroidales genetic markers for human and animal microbial source tracking in tropical agricultural watersheds. Environ Pollut. 2018;236:100-10.

14. Fan LH, Shuai JB, Zeng RX, Mo HF, Wang SH, Zhang XF, et al. Validation and application of quantitative PCR assays using host-specific Bacteroidales genetic markers for swine fecal pollution tracking. Environ Pollut. 2017;231:1569-77.

15. Zapata HJ, Quagliarello VJ. The microbiota and microbiome in aging: Potential implications in health and age-related diseases. J Am Geriatr Soc. 2015;63(4):776-81. 
16. Zhang Q, Ying G, Pan C, Liu Y, Zhao J. Comprehensive evaluation of antibiotics emission and fate in the river basins of China: source analysis, multimedia modeling, and linkage to bacterial resistance. Environ Sci Technol. 2015;49(11):6772-82

17. Falagas ME, Kasiakou SK. Colistin: the revival of polymyxins for the management of multidrugresistant gram-negative bacterial infections. Clin Infect Dis. 2005;40(9):1333-41.

18. Pallav K, Dowd SE, Villafuerte J, Yang X, Kabbani T, Hansen J, et al. Effects of polysaccharopeptide from Trametes versicolor and amoxicillin on the gut microbiome of healthy volunteers: a randomized clinical trial. Gut Microbes. 2014;5(4):458-67.

19. Oh B, Kim BS, Kim JW, Kim JS, Koh SJ, Kim BG, et al. The effect of probiotics on gut microbiota during the Helicobacter pylori eradication: randomized controlled trial. Helicobacter. 2016;21(3):16574.

20. Reijnders D, Goossens GH, Hermes GD, Neis EP, van der Beek CM, Most J, et al. Effects of gut microbiota manipulation by antibiotics on host metabolism in obese humans: a randomized doubleblind placebo-controlled trial. Cell Metab. 2016;24(1):63-74.

21. Zaura E, Brandt BW, Teixeira de Mattos MJ, Buijs MJ, Caspers MP, Rashid MU, et al. Same exposure but two radically different responses to antibiotics: Resilience of the salivary microbiome versus long-term microbial shifts in feces. MBio. 2015;6(6):e01693-15.

22. Jeong SH, Song YK, Cho JH. Risk assessment of ciprofloxacin, flavomycin, olaquindox and colistin sulfate based on microbiological impact on human gut biota. Regul Toxicol Pharmacol. 2009;53(3):209-16.

23. Dimitriou V, Biehl LM, Hamprecht A, Vogel W, Dorfel D, Peter S, et al. Controlling intestinal colonization of high-risk haematology patients with ESBL-producing Enterobacteriaceae: a randomized, placebo-controlled, multicentre, Phase II trial (CLEAR). J Antimicrob Chemother. 2019;74(7):2065-74.

24. Huttner BD, de Lastours V, Wassenberg M, Maharshak N, Mauris A, Galperine T, et al. A 5-day course of oral antibiotics followed by faecal transplantation to eradicate carriage of multidrug-resistant Enterobacteriaceae: a randomized clinical trial. Clin Microbiol Infect. 2019;25(7):830-8.

25. Ghiselli R, Giacometti A, Cirioni O, Mocchegiani F, Viticchi C, Scalise G, et al. Cationic peptides combined with betalactams reduce mortality from peritonitis in experimental rat model. J Surg Res. 2002;108(1):107-11.

26. Soler C, Goossens T, Bermejo A, Migura-García L, Cusco A, Francino O, et al. Digestive microbiota is different in pigs receiving antimicrobials or a feed additive during the nursery period. PLoS One. 2018;13:e0197353.

27. Sokół I, Tokarzewski S, Bobrek K, Gaweł A. The effect of the administration of different antimicrobial formulations on the fungal infestation of the gastrointestinal tract in turkeys. Pak Vet $\mathrm{J}$. 2017;37:475-9.

28. Marzorati M, Van de Wiele T. An advanced in vitro technology platform to study the mechanism of action of prebiotics and probiotics in the gastrointestinal tract. J Clin Gastroenterol. 2016;50:S124- 
S5.

29. Ichim TE, Kesari S, Shafer K. Protection from chemotherapy- and antibiotic-mediated dysbiosis of the gut microbiota by a probiotic with digestive enzymes supplement. Oncotarget. 2018;9(56):30919-35.

30. Marzorati M, Vilchez-Vargas R, Bussche JV, Truchado P, Jauregui R, El Hage RA, et al. High-fiber and high-protein diets shape different gut microbial communities, which ecologically behave similarly under stress conditions, as shown in a gastrointestinal simulator. Mol Nutr Food Res. 2017;61(1):1600150.

31. Van den Abbeele P, Roos S, Eeckhaut V, MacKenzie DA, Derde M, Verstraete W, et al. Incorporating a mucosal environment in a dynamic gut model results in a more representative colonization by Lactobacilli. Microb Biotechnol. 2012;5(1):106-15.

32. El Hage R, Hernandez-Sanabria E, Calatayud Arroyo M, Props R, Van de Wiele T. Propionateproducing consortium restores antibiotic-induced dysbiosis in a dynamic in vitro model of the human intestinal microbial ecosystem. Front Microbiol. 2019;10:1206.

33. Yu HY, Wu B, Zhang XX, Liu S, Yu J, Cheng SP, et al. Arsenic metabolism and toxicity influenced by ferric iron in simulated gastrointestinal tract and the roles of gut microbiota. Environ Sci Technol. 2016;50(13):7189-97.

34. Gao Z, Guo B, Gao R, Zhu Q, Qin H. Microbiota disbiosis is associated with colorectal cancer. Front Microbiol. 2015;6:1-9.

35. Li S, Zhang C, Gu Y, Chen L, Ou S, Wang Y, et al. Lean rats gained more body weight than obese ones from a high-fibre diet. Br J Nutr. 2015;114(8):1188-94.

36. MacPherson CW, Mathieu O, Tremblay J, Champagne J, Nantel A, Girard SA, et al. Gut bacterial microbiota and its resistome rapidly recover to basal state levels after short-term amoxicillinclavulanic acid treatment in healthy adults. Sci Rep. 2018;8(1):11192.

37. Perez-Cobas AE, Artacho A, Knecht H, Ferrus ML, Friedrichs A, Ott SJ, et al. Differential effects of antibiotic therapy on the structure and function of human gut microbiota. PLoS One. 2013;8(11):e80201.

38. Barraud O, Peyre M, Couve-Deacon E, Chainier D, Bahans C, Guigonis V, et al. Antibiotic resistance acquisition in the first week of life. Front Microbiol. 2018;9:1467.

39. Buelow E, Gonzalez TB, Versluis D, Oostdijk EA, Ogilvie LA, van Mourik MS, et al. Effects of selective digestive decontamination (SDD) on the gut resistome. J Antimicrob Chemother. 2014;69(8):221523.

40. Parish T. Steps to address anti-microbial drug resistance in today's drug discovery. Expert Opin Drug Discov. 2019;14(2):91-4.

41. Tangden T. Combination antibiotic therapy for multidrug-resistant Gram-negative bacteria. Ups J Med Sci. 2014;119(2):149-53.

42. Patel M, Bowe W, Heughebaert C, Shalita A. The development of antimicrobial resistance due to the antibiotic treatment of acne vulgaris: A review. Journal of drugs in dermatology : JDD. 2010;9:65564. 
43. Bliziotis IA, Samonis G, Vardakas KZ, Chrysanthopoulou S, Falagas ME. Effect of aminoglycoside and beta-lactam combination therapy versus beta-lactam monotherapy on the emergence of antimicrobial resistance: a meta-analysis of randomized, controlled trials. Clin Infect Dis. 2005;41(2):149-58.

44. Akins RL, Barber KE, Palmer KL. Pronounced heterogeneity observed in high-level daptomycinresistant viridans group streptococci. J Glob Antimicrob Resist. 2016;7:159-66.

45. Vestergaard M, Paulander W, Marvig RL, Clasen J, Jochumsen N, Molin S, et al. Antibiotic combination therapy can select for broad-spectrum multidrug resistance in Pseudomonas aeruginosa. Int J Antimicrob Agents. 2016;47(1):48-55.

46. Schrag SJ, Pena C, Fernandez J, Sanchez J, Gomez V, Perez E, et al. Effect of short-course, high-dose amoxicillin therapy on resistant pneumococcal carriage: a randomized trial. JAMA. 2001;286(1):4956.

47. Ubeda C, Pamer EG. Antibiotics, microbiota, and immune defense. Trends Immunol. 2012;33(9):45966.

48. Oh B, Kim JW, Kim BS. Changes in the functional potential of the gut microbiome following probiotic supplementation during Helicobacter pylori treatment. Helicobacter. 2016;21(6):493-503.

49. Forssten S, Evans M, Wilson D, Ouwehand AC. Influence of a probiotic mixture on antibiotic induced microbiota disturbances. World J Gastroenterol. 2014;20(33):11878-85.

50. Morley VJ, Woods RJ, Read AF. Bystander selection for antimicrobial resistance: Implications for patient health. Trends Microbiol. 2019;27(10):864-77.

51. Oh J, Patel J, Park HB, Crawford JM. beta-Lactam biotransformations activate innate immunity. J Org Chem. 2018;83(13):7173-9.

52. Kabbani TA, Pallav K, Dowd SE, Villafuerte-Galvez J, Vanga RR, Castillo NE, et al. Prospective randomized controlled study on the effects of Saccharomyces boulardii CNCM I-745 and amoxicillinclavulanate or the combination on the gut microbiota of healthy volunteers. Gut Microbes. 2017;8(1):17-32.

53. Lin Y-T, Liu C-J, Yeh Y-C, Chen T-J, Fung C-P. Ampicillin and amoxicillin use and the risk of Klebsiella pneumoniae liver abscess in Taiwan. J Infect Dis. 2013;208(2):211-7.

54. Jess T, Morgen CS, Harpsoe MC, Sorensen TIA, Ajslev TA, Antvorskov JC, et al. Antibiotic use during pregnancy and childhood overweight: A population-based nationwide cohort study. Sci Rep. 2019;9.

55. Ladirat SE, Schoterman MHC, Rahaoui H, Mars M, Schuren FHJ, Gruppen H, et al. Exploring the effects of galacto-oligosaccharides on the gut microbiota of healthy adults receiving amoxicillin treatment. Br J Nutr. 2014;112(4):536-46.

56. Vrieze A, Out C, Jonker L, Holleman F, Dallinga-Thie GM, Ackermans MT, et al. Differential effects of antibiotics on intestinal microbiota composition and insulin resistance in obese humans; a randomised controlled trial. Diabetologia. 2012;55:S265-S.

57. Zhang J, Haines C, Watson AJM, Hart AR, Platt MJ, Pardoll DM, et al. Oral antibiotic use and risk of colorectal cancer in the United Kingdom, 1989-2012: a matched case-control study. Gut. 
2019;68(11):1971-8.

58. Simon K, Verwoolde MB, Zhang J, Smidt H, Reilingh GdV, Kemp B, et al. Long-term effects of early life microbiota disturbance on adaptive immunity in laying hens. Poult Sci. 2016;95(7):1543-54.

59. Candon S, Perez-Arroyo A, Marquet C, Valette F, Foray AP, Pelletier B, et al. Antibiotics in early life alter the gut microbiome and increase disease incidence in a spontaneous mouse model of autoimmune insulin-dependent diabetes. PLoS One. 2015;10(5):e0125448.

60. Neveling DP, van Emmenes L, Ahire JJ, Pieterse E, Smith C, Dicks LMT. Safety assessment of antibiotic and probiotic feed additives for Gallus gallus domesticus. Sci Rep. 2017;7(1):12767.

61. Cao G, Tao F, Hu Y, Li Z, Zhang Y, Deng B, et al. Positive effects of a Clostridium butyricum-based compound probiotic on growth performance, immune responses, intestinal morphology, hypothalamic neurotransmitters, and colonic microbiota in weaned piglets. Food Funct. 2019;10(5):2926-34.

62. Wang H, Zhang W, Zuo L, Dong J, Zhu W, Li Y, et al. Intestinal dysbacteriosis contributes to decreased intestinal mucosal barrier function and increased bacterial translocation. Lett Appl Microbiol. 2014;58(4):384-92.

63. Qureshi ZA, Paterson DL, Potoski BA, Kilayko MC, Sandovsky G, Sordillo E, et al. Treatment outcome of bacteremia due to KPC-producing Klebsiella pneumoniae: Superiority of combination antimicrobial regimens. Antimicrob Agents Chemother. 2012;56(4):2108-13.

64. Szuster-Ciesielska A, Urban-Chmiel R, Wernicki A, Mascaron L, Wasak M, Bousquet E. Evaluation of the ability of colistin, amoxicillin (components of Potencil $($ )), and fluoroquinolones to attenuate bacterial endotoxin- and Shiga exotoxin-mediated cytotoxicity-In vitro studies. J Vet Pharmacol Ther. 2019;42(1):85-103.

65. Tamma PD, Cosgrove SE, Maragakis LL. Combination therapy for treatment of infections with gramnegative bacteria. Clin Microbiol Rev. 2012;25(3):450-70.

66. Paul M, Lador A, Grozinsky-Glasberg S, Leibovici L. Beta lactam antibiotic monotherapy versus beta lactamaminoglycoside antibiotic combination therapy for sepsis. Cochrane Database of Systematic Reviews. 2014(1).

67. Barc MC, Charrin-Sarnel C, Rochet V, Bourlioux F, Sandre C, Boureau H, et al. Molecular analysis of the digestive microbiota in a gnotobiotic mouse model during antibiotic treatment: influence of Saccharomyces boulardii. Anaerobe. 2008;14(4):229-33.

68. Collignon A, Sandre C, Barc MC. Saccharomyces boulardii modulates dendritic cell properties and intestinal microbiota disruption after antibiotic treatment. Gastroenterol Clin Biol. 2010;34:S71-S8.

69. Vrieze A, Out C, Fuentes S, Jonker L, Reuling I, Kootte RS, et al. Impact of oral vancomycin on gut microbiota, bile acid metabolism, and insulin sensitivity. J Hepatol. 2014;60(4):824-31.

70. Kohler T, Michea-Hamzehpour M, Henze U, Gotoh N, Curty LK, Pechere JC. Characterization of MexEMexF-OprN, a positively regulated multidrug efflux system of Pseudomonas aeruginosa. Mol Microbiol. 1997;23(2):345-54. 
71. Roy Chowdhury P, Scott MJ, Djordjevic SP. Genomic islands 1 and 2 carry multiple antibiotic resistance genes in Pseudomonas aeruginosa ST235, ST253, ST111 and ST175 and are globally dispersed. J Antimicrob Chemother. 2017;72(2):620-2.

72. Kim MJ, Ku S, Kim SY, Lee HH, Jin H, Kang S, et al. Safety evaluations of Bifidobacterium bifidum BGN4 and Bifidobacterium longum BORI. Int J Mol Sci. 2018;19(5).

73. Luo $C$, Hang $X$, Liu $X$, Zhang $M$, Yang $X$, Yang H. Detection of erm $(X)$-mediated antibiotic resistance in Bifidobacterium longum subsp. longum. Ann Microbiol. 2015;65(4):1985-91.

74. Chen J, Chen Y, Hu P, Zhou T, Xu X, Pei X. Risk assessment of infected children with Pseudomonas aeruginosa pneumonia by combining host and pathogen predictors. Infect Genet Evol. 2018;57:82-7.

75. Meradji S, Barguigua A, Bentakouk MC, Nayme K, Zerouali K, Mazouz D, et al. Epidemiology and virulence of VIM-4 metallo-beta-lactamase-producing Pseudomonas aeruginosa isolated from burn patients in eastern Algeria. Burns. 2016;42(4):906-18.

76. Oliver A, Mulet X, Lopez-Causape C, Juan C. The increasing threat of Pseudomonas aeruginosa highrisk clones. Drug Resist Updat. 2015;21-22:41-59.

77. Souza Lopes AC, Rodrigues JF, Cabral AB, da Silva ME, Leal NC, da Silveira VM, et al. Occurrence and analysis of irp2 virulence gene in isolates of Klebsiella pneumoniae and Enterobacterspp. from microbiota and hospital and community-acquired infections. Microb Pathog. 2016;96:15-9.

78. Laurens $C$, Jean-Pierre H, Licznar-Fajardo P, Hantova S, Godreuil S, Martinez O, et al. Transmission of IMI-2 carbapenemase-producing Enterobacteriaceae from river water to human. J Glob Antimicrob Resist. 2018;15:88-92.

79. Shankar V, Hamilton MJ, Khoruts A, Kilburn A, Unno T, Paliy O, et al. Species and genus level resolution analysis of gut microbiota in Clostridium difficile patients following fecal microbiota transplantation. Microbiome. 2014;2:13.

80. Hammer K, Stoessel A, Justo JA, Bookstaver B, Kohn J, Derrick C, et al. Association between chronic hemodialysis and bloodstream infections caused by chromosomally mediated AmpC-producing Enterobacteriaceae. Am J Infect Control. 2016;44(12):1611-6

81. Khaertynov HS, Semenova DS, Sushnikov KV. Clinical and epidemiological features of acute intestinal infections with hemorragic colitis in children Kazan Medical Journal. 2013;94(2):208-11.

82. Pesola J, Hakalehto E. Enterobacterial microflora in infancy - a case study with enhanced enrichment. Indian J Pediatr. 2011;78(5):562-8.

83. Zhang L, Wu Y-N, Chen T, Ren C-H, Li X, Liu G-X. Relationship between intestinal microbial dysbiosis and primary liver cancer. Hepatobiliary Pancreat Dis Int. 2019;18(2):149-57.

84. Li N, Wang X, Sun C, Wu X, Lu M, Si Y, et al. Change of intestinal microbiota in cerebral ischemic stroke patients. BMC Microbiol. 2019;19(1):191.

85. Zhao X, Chen Z, Yin Y, Li X. Effects of polysaccharide from Physalis alkekengi var. francheti on liver injury and intestinal microflora in type-2 diabetic mice. Pharm Biol. 2017;55(1):2020-5. 
86. Duvallet C, Gibbons SM, Gurry T, Irizarry RA, Alm EJ. Meta-analysis of gut microbiome studies identifies disease-specific and shared responses. Nat Commun. 2017;8(1):1784.

87. Al Yazidi LS, McMullan B, Kohan S, Palasanthiran P. Persistent gram-negative neurosurgical meningitis in a neonate, successfully treated with intraventricular colistin: Case report and review of the literature. Pediatr Infect Dis J. 2018;37(3):E79-E81.

88. Van den Abbeele P, Belzer C, Goossens M, Kleerebezem M, De Vos WM, Thas O, et al. Butyrateproducing Clostridium clusterXIVa species specifically colonize mucins in an in vitro gut model. ISME J. 2013;7(5):949-61.

89. Caporaso JG, Kuczynski J, Stombaugh J, Bittinger K, Bushman FD, Costello EK, et al. QIIME allows analysis of high-throughput community sequencing data. Nat Methods. 2010;7(5):335-6.

90. Wang Q, Garrity GM, Tiedje JM, Cole JR. Naive Bayesian classifier for rapid assignment of rRNA sequences into the new bacterial taxonomy. Appl Environ Microbiol. 2007;73(16):5261-7.

91. Liu L, Wang Q, Lin H, Das R, Wang S, Qi H, et al. Amoxicillin Increased Functional Pathway Genes and Beta-Lactam Resistance Genes by Pathogens Bloomed in Intestinal Microbiota Using a Simulator of the Human Intestinal Microbial Ecosystem. Front Microbiol. 2020;11(1213).

92. Liu L, Wang Q, Wu X, Qi H, Das R, Lin H, et al. Vancomycin exposure caused opportunistic pathogens bloom in intestinal microbiome by simulator of the human intestinal microbial ecosystem (SHIME). Environ Pollut. 2020:114399.

93. Segata N, Izard J, Waldron L, Gevers D, Miropolsky L, Garrett WS, et al. Metagenomic biomarker discovery and explanation. Genome Biol. 2011;12(6):R60.

94. Langille MGI, Zaneveld J, Caporaso JG, McDonald D, Knights D, Reyes JA, et al. Predictive functional profiling of microbial communities using 16S rRNA marker gene sequences. Nat Biotechnol. 2013;31(9):814-21.

95. Hu Y, Yang X, Qin J, Lu N, Cheng G, Wu N, et al. Metagenome-wide analysis of antibiotic resistance genes in a large cohort of human gut microbiota. Nat Commun. 2013;4:2151-249.

\section{Figures}




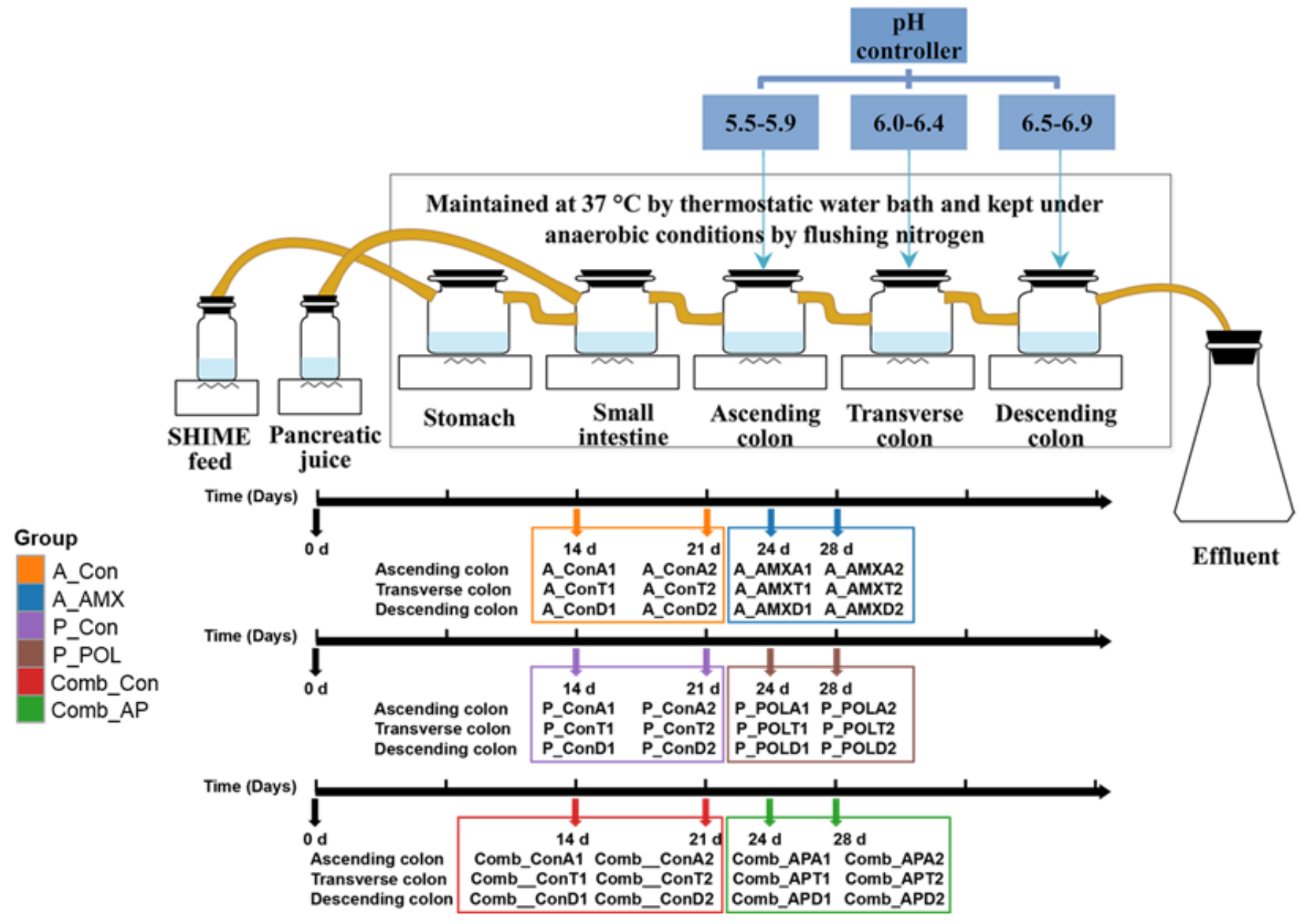

Figure 1

Schematic of designed SHIME model and sampling time points setup.

(a)

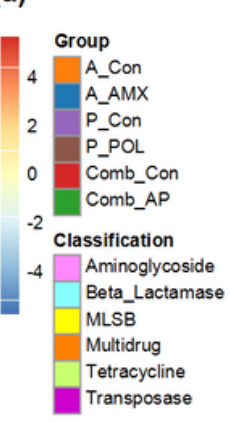

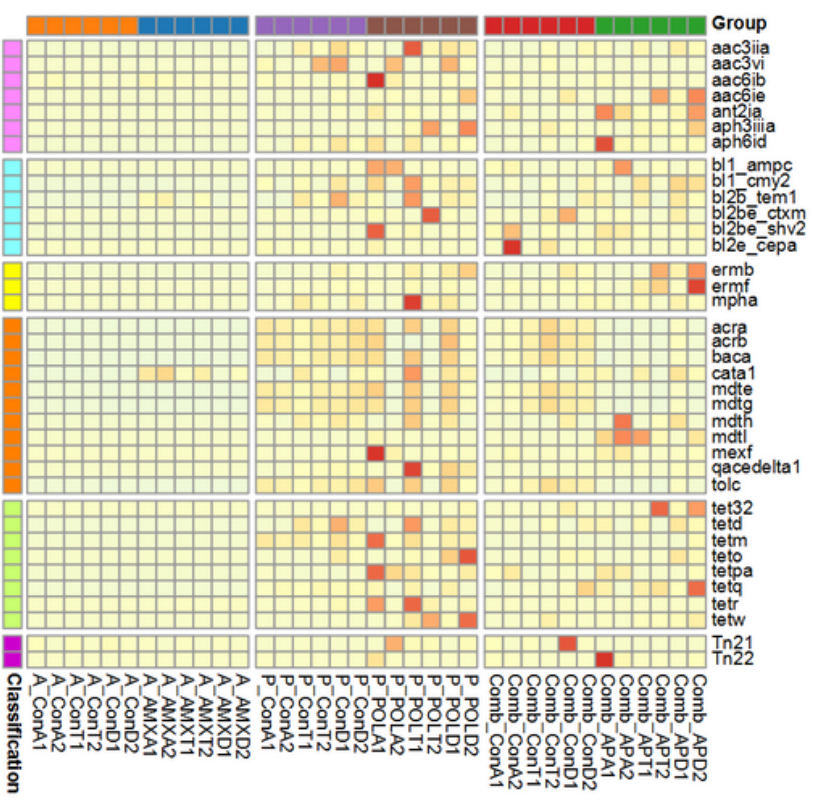

(b)

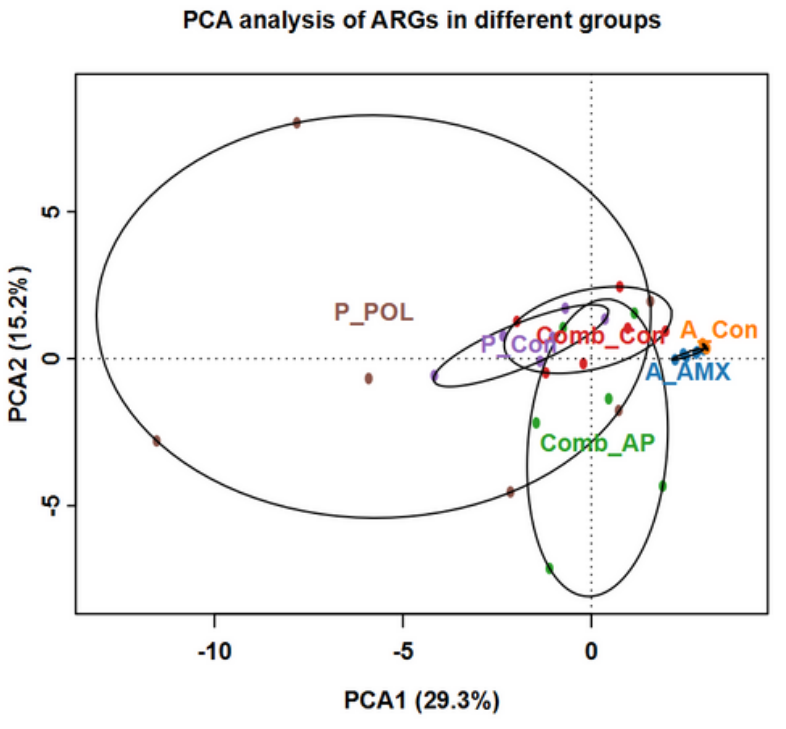


Heatmap (a) and PCA (b) analysis of antibiotic resistance genes (ARGs) in the six groups. Heatmap colors reflect relative abundance of ARGs from low (blue) to high (red).

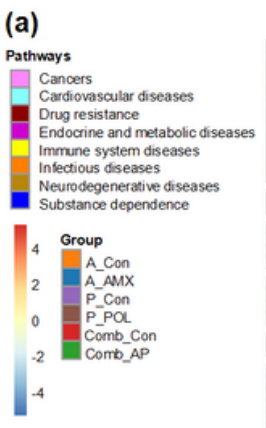

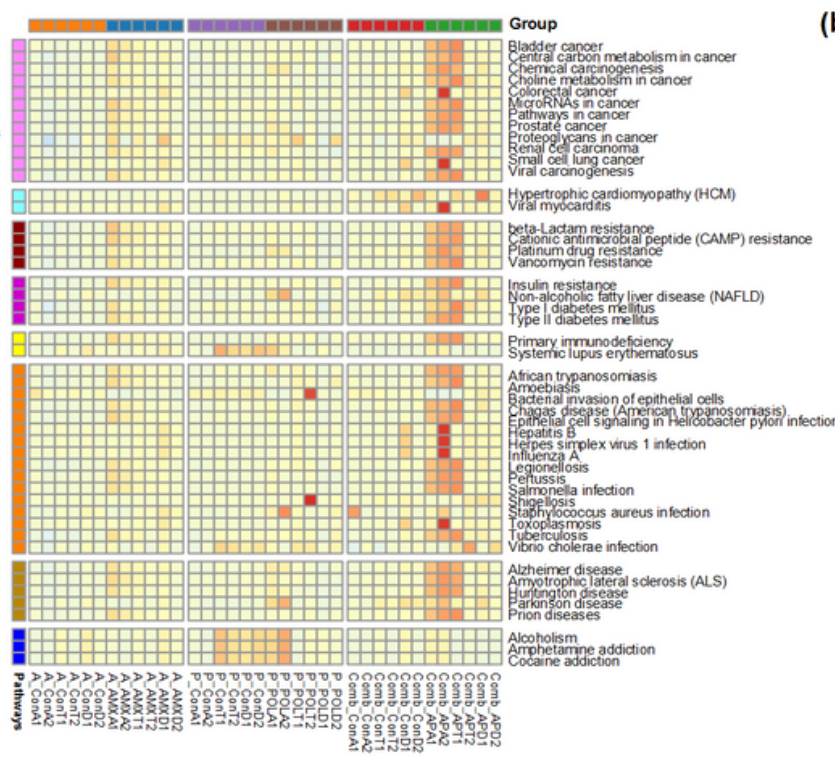

(b)

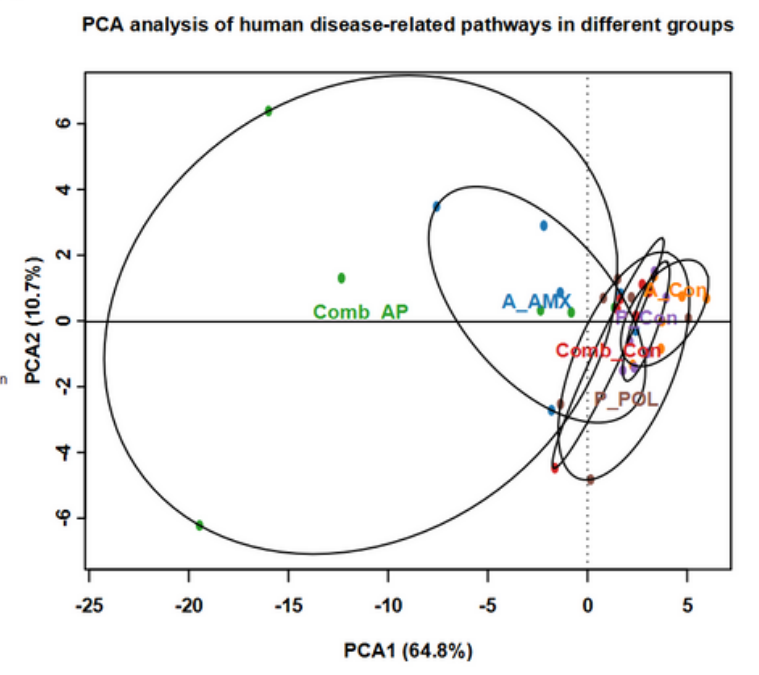

Figure 3

Heatmap (a) and PCA (b) analysis of human disease-related pathways in the six groups. Heatmap colors reflect gene numbers of human disease-related pathways from low (blue) to high (red).

(a)

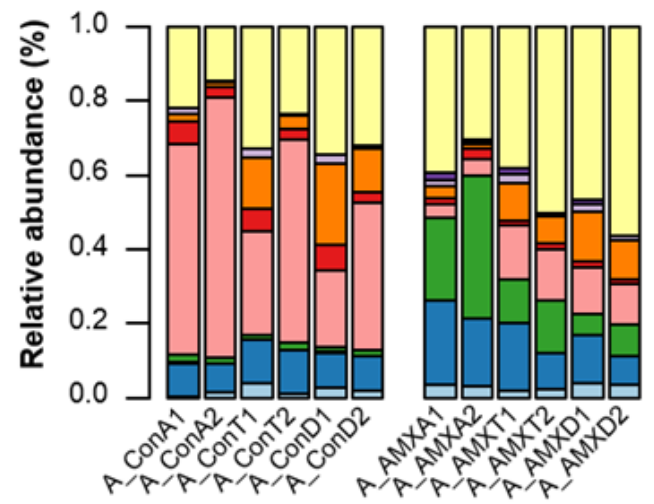

(c)

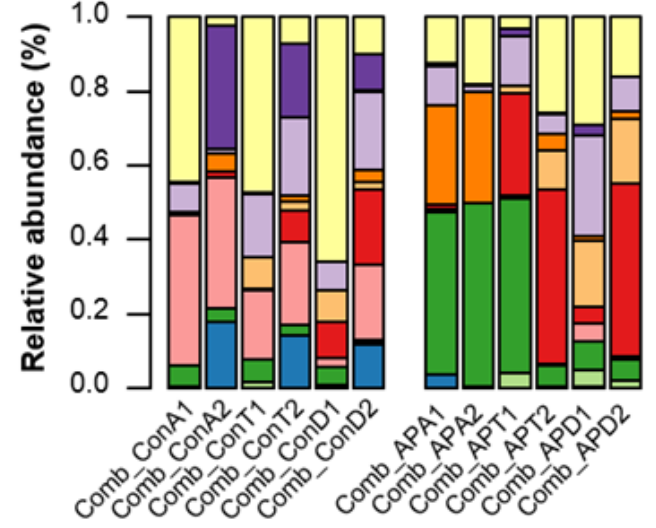

(b)

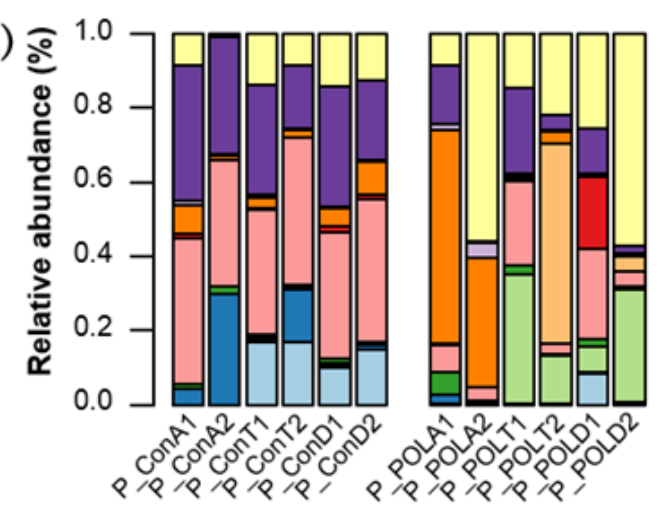

(d)

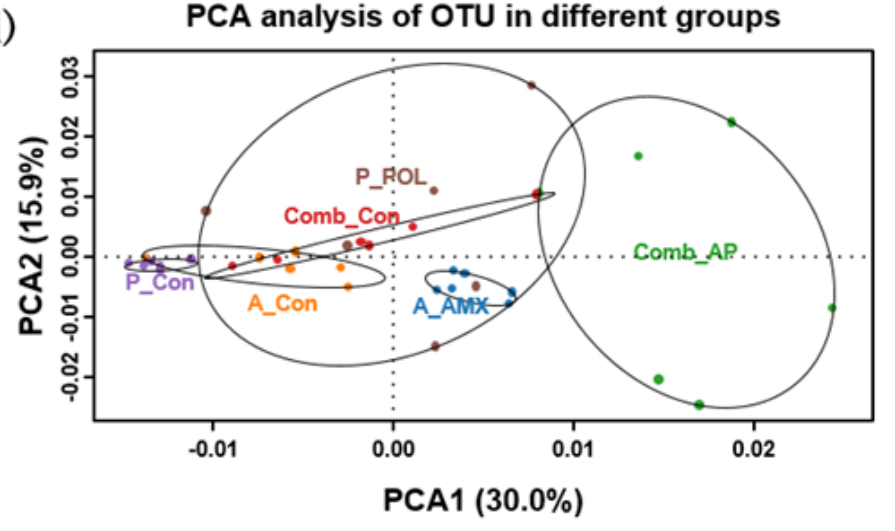

Figure 4 
Composition of microbial community at genus level in groups A_Con and A_AMX (a), P_Con and P_POL (b), Comb_Con and Comb_AP (c), and PCA analysis of OTUs in the six groups (d).

(a)

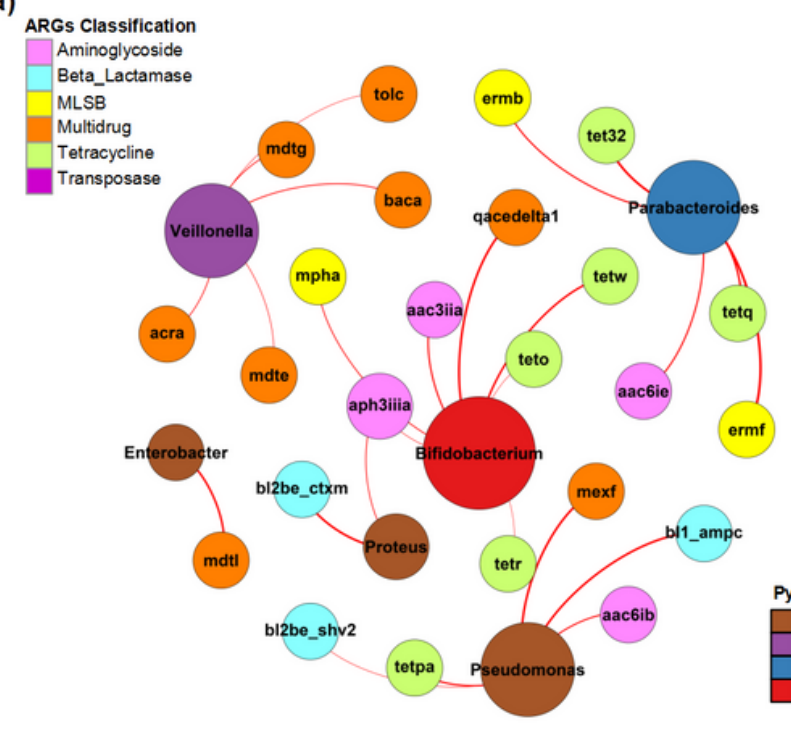

(b)

Pathways classification

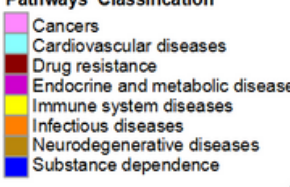

Cancers
Crdiovascular diseases
Eng resistance
Endocrine and metabolic diseases

Immune system diseases

Nubstance dependence
Substive
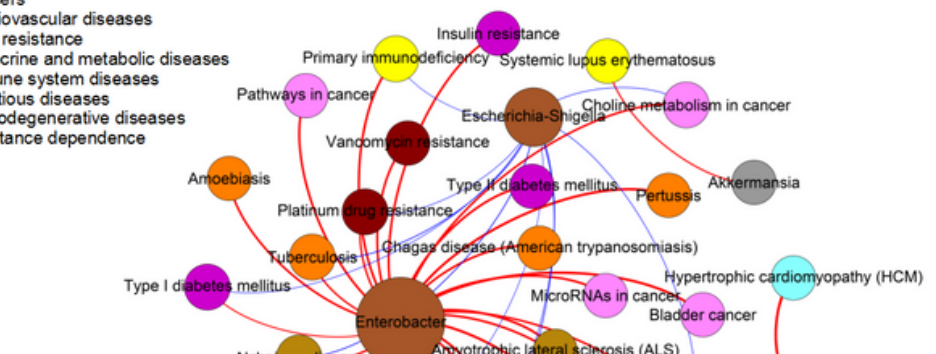

ylum Classification Proteobacteria Firmicutes Bacteroidetes Actinobacteria

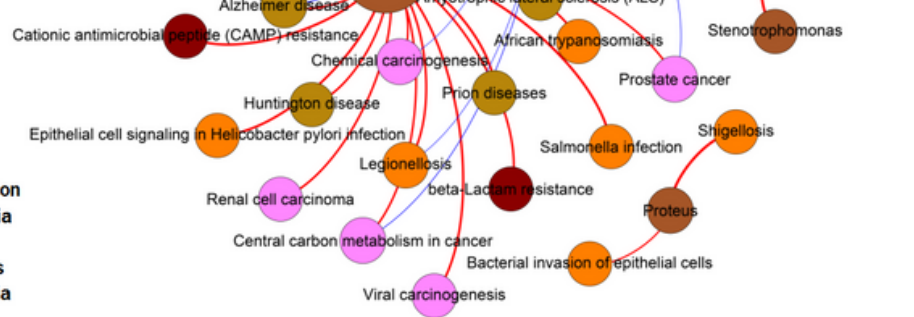

Figure 5

Network analysis revealing the co-occurrence patterns between microbial taxa and ARG subtypes (a) or human disease-related pathways (b). The nodes in Network were colored according to ARG types, human disease-related pathways types, and microbial phylum. The edges were colored according to positive (red) or negative (blue) correlation. A connection represents strong and significant $(P$ value $<0.05, r>0.6)$ correlation. The size of each node is proportional to the number of connections, that is, degree.

\section{Supplementary Files}

This is a list of supplementary files associated with this preprint. Click to download.

- Additionalfile1.doc

- Additionalfile2.xls

- GraphicalAbstract.doc 